
\title{
$C^{-1} \begin{aligned} & \text { CONGRESO } \\ & \text { INTERNACIONAL }\end{aligned}$ SOBRE FOTOGRAFÍA
}

Congreso Internacional sobre Fotografía

UPV, 5 y 6 octubre 2017

Doi: http://dx.doi.org/10.4995/CIFo17.2017.6749

ISBN: 978-84-9048-604-7

\section{La fotografía en la docencia del área de Biblioteconomía y Documentación en las universidades públicas madrileñas. Actividades del Grupo Fotodoc}

Juan Miguel Sánchez Vigil ${ }^{a}$, María Olivera Zaldua ${ }^{b}$ y Antonia Salvador Benítez

${ }^{a}$ Grupo de Investigación Fotodoc, Facultad de Ciencias de la Documentación, Universidad Complutense de Madrid, jmvigil@ucm.es, ${ }^{\mathrm{b}}$ Grupo de Investigación Fotodoc, Facultad de Ciencias de la Documentación, Universidad Complutense de Madrid, molivera@ucm.es y ${ }^{\mathrm{c}}$ Grupo de Investigación Fotodoc, Facultad de Ciencias de la Documentación, Universidad Complutense de Madrid, asalvado@ucm.es.

\begin{abstract}
Teaching photography in relation with Library Science and Documentation in Madrid's public universities began in the old Escuela de Biblioteconomia y Documentation of the Universidad Complutense of Madrid at the end of the decade of the 90s through courses of content analysis. As regards the rest of the universities of the Comunidad de Madrid, in the area under study, only the Universidad Carlos III has carried out extensive work in the Faculty of Humanities, Communication and Documentation through its Image, Culture and Technology Seminars, which are now inactive. When the academic degree in Documentation in the Faculty of Journalism was approved, the optional course of "Photographic Documentation" was created. Afterwards, the course of Photographic and Iconographic Documentation was included in the Masters on Media Management of the UCM. During the decade of 2000-2010, the photograph considered as a document took on greater specific importance and as of 2010, the Fotodoc Work Group was created in the UCM, which was the origin of the Research Group of the same name, officially recognized in June of 2016. This intensive activity, linked to teaching at Undergraduate and Masters levels, led to the creation of the International Photographic Documentation Congresses in which institutions from Mexico, Portugal, Brazil and Spain have participated to date. This article covers the status of Photography as a course in the Madrid universities, namely in the Faculty of Documentation of the UCM, as well as the work carried out by the Fotodoc Research.
\end{abstract}

Keywords: Photography teaching, Research groups, Fotodoc Seminars, Innovation teaching,Photography Congress.

\footnotetext{
Resumen

La docencia sobre fotografia relacionada con la Biblioteconomía y Documentación en las universidades públicas madrileñas surgió en la antigua Escuela de Biblioteconomía y Documentación de la Universidad Complutense de Madrid a finales de los años 90 mediante cursos de análisis de contenidos. Por lo que respecta al resto de universidades de la Comunidad de Madrid, en el área que tratamos solo la Universidad Carlos III ha desarrollado la Facultad de Humanidades, Comunicación y Documentación una intensa labor expuesta a través de las
} 
La fotografia en la docencia del área de Biblioteconomía y Documentación en las universidades públicas madrileñas. Actividades del Grupo Fotodoc

Jornadas Imagen, Cultura y Tecnología, hoy inactivas. Al aprobarse la Licenciatura en Documentación en la Facultad de Ciencias de la Información, se creó la asignatura optativa "Documentación Fotográfica". Posteriormente se incluyó la asignatura Documentación Fotográfica e Iconográfica en el Master de Gestión de la Información de la UCM. Durante la década 2000-2010 la fotografía como documento fue cogiendo peso específico y a partir de este último año 2010 se creó en la UCM el Grupo de Trabajo Fotodoc, germen del Grupo de Investigación del mismo nombre, reconocido oficialmente en junio de 2016. Fruto de su intensa actividad, vinculada a la docencia en Grado y Máster, es la creación de los Congresos Internacionales de Documentación Fotográfica en el que han participado hasta la fecha instituciones de México, Portugal, Brasil y España. Se expone en este artículo la situación de la Fotografia cono docencia en las universidades madrileñas, concretamente en la Facultad de Documentación de la UCM, así como las tareas desarrolladas por el Grupo de Investigación Fotodoc.

Palabras clave: Docencia fotográfica, Grupo de Investigación, Jornadas Fotodoc, Innovación docente, Congresos de fotografia.

\section{Introducción}

La docencia sobre fotografía se remonta en la Universidad española a finales del siglo XIX, propiciada por el asociacionismo y por su aplicación a la ciencia. El intento por llevar la fotografía a la Universidad tiene antecedentes en 1883, con la noticia publicada en el diario político, literario y mercantil La Dinastía el 29 de noviembre de ese año, donde se informa sobre la "posible" fundación de la Sociedad Catalana de Fotografía en la Facultad de Ciencias de la Universidad de Barcelona, al objeto de llevar a cabo estudios teóricos y prácticos de fotografía y galvanoplastia. Una semana después, el 4 de diciembre, el mismo diario informaba que, "como se había anunciado, quedaba constituida la Sociedad Española de Aficionados a la Fotografía, es decir con un ámbito de actuación estatal. Esta agrupación tuvo poco recorrido, ya que un periodista que firmó con una "X" mayúscula para ocultar su identidad, publicó en La Fotografía de Barcelona (1886, p. 324) el artículo "Necesidad de una sociedad fotográfica española", en el que comentó que mientras fuera se creaban cátedras, en España sucedía todo lo contrario. Por dos veces se había intentado la formación de una Sociedad Fotográfica Española, en la primera se empezó con gran interés, se creó un fondo para los trabajos preliminares y cuando parecía que todo marchaba se produjo el enfrentamiento entre aficionados y amateurs; en la segunda se llegaron a redactar estatutos pero no hubo medios económicos.

En 1900, el Ejército dedicó parte del programa de formación de oficiales al conocimiento y manejo de las cámaras fotográficas, con una asignatura obligatoria para los alumnos de la Academia de Artillería de Segovia. Dentro del programa de instrucción, en la sección "Servicios especiales" se consideraba imprescindible conocer el telégrafo Morse, el teléfono Ader de campaña, el aparato magnético de Siemens y la cámara Kodak de campaña, con las que se obtenían "negativas en papel" (El Correo militar, 9 de julio de 1890). En el apogeo pictorialista, fueron aprobados los primeros estudios oficiales de fotografia en la Escuela Superior de Guerra, por Real Decreto de 31 de mayo de 1904, aunque, con carácter privado, ya se impartían clases en las agrupaciones de aficionados.

Los profesionales fueron conscientes de la necesidad de difundir la fotografía a través de la enseñanza reglada y en la Asamblea Nacional celebrada en Valencia en 1908 acordaron en la conclusión novena de la Memoria final 
que se crearan escuelas teóricas y prácticas con sede en la localidad que más tributara, y con sucursales. La propuesta no pudo llevarse a cabo por la desconfianza entre los profesionales (La Fotografia, enero de 1909).

La Escuela de Caminos incorporó en 1910 una asignatura sobre el uso y manejo de los aparatos fotográficos, el revelado y el positivado. El responsable fue el ingeniero Antonio Sonier, experto en estereoscopia. El sistema de aprendizaje era moderno, poniendo en manos de los alumnos el aparato y las placas para obtener vistas. En 1913 comenzaron las clases de fotografía en la Escuela de Artes Gráficas de Madrid, si bien los cursos se habían aprobado en diciembre de 1910, con talleres de heliograbado, litografía, fotograbado, fotografía y encuadernación. La primera Escuela Oficial de Fotografía se fundaría en Granada el año 1916, dirigida por Manuel Torres Molina, uno de los grandes autores del primer tercio del siglo XX.

A partir de entonces la enseñanza de la fotografía se fue incorporando a las escuelas de artes y oficios, siempre con un sesgo hacia los aspectos técnicos, fundamentalmente hacia la toma y al conocimiento de las cámaras y materiales empleados para la aplicación. Desde los años treinta, ya con las vanguardias haciendo de la fotografía uno de los elementos claves en la creación, proliferaron la escuelas privadas, si bien en la enseñanza reglada continuó el vacío. Hasta la década de los setenta no se contempló la incorporación de la foto a los estudios universitarios, y ello gracias a la creación de facultades dedicadas al estudio de la Información, Comunicación y, posteriormente, la Documentación.

\section{Docencia de la Fotografía en Documentación}

Dando un salto en el tiempo para tratar sobre el tema que nos ocupa, es decir sobre la docencia relacionada con los estudios de Documentación en las universidades públicas madrileñas, hemos de indicar que se aplicaron por primera vez en la antigua Escuela de Biblioteconomía y Documentación de la Universidad Complutense de Madrid a finales de los años 90 (Fig. 1), mediante cursos (no reglados) de análisis de contenidos, historia y uso y aplicación de la fotografía en prensa y edición. Posteriormente, al aprobarse la Licenciatura en Documentación en 1996, como una de las ramas de los estudios en Ciencias de la Información impartidos en la Facultad de Ciencias de la Información de la Universidad Complutense, se creó la asignatura optativa "Documentación Fotográfica", ofertada a los alumnos de cuarto y quinto curso, e impartida por Juan Miguel Sánchez Vigil. En relación a esta decisión es importante destacar la publicación del primer Manual de Documentación Fotográfica (Síntesis, 1999), coordinado por Félix del Valle Gastaminza, y en el que colaboraron los profesores Carmen Agustín, Adelina Clausó, Ángel María Fuentes, Francisco Javier García Marco, Antonio Hernández, Luis Fernando Ramos, Jesús Robledano y Juan Miguel Sánchez Vigil.

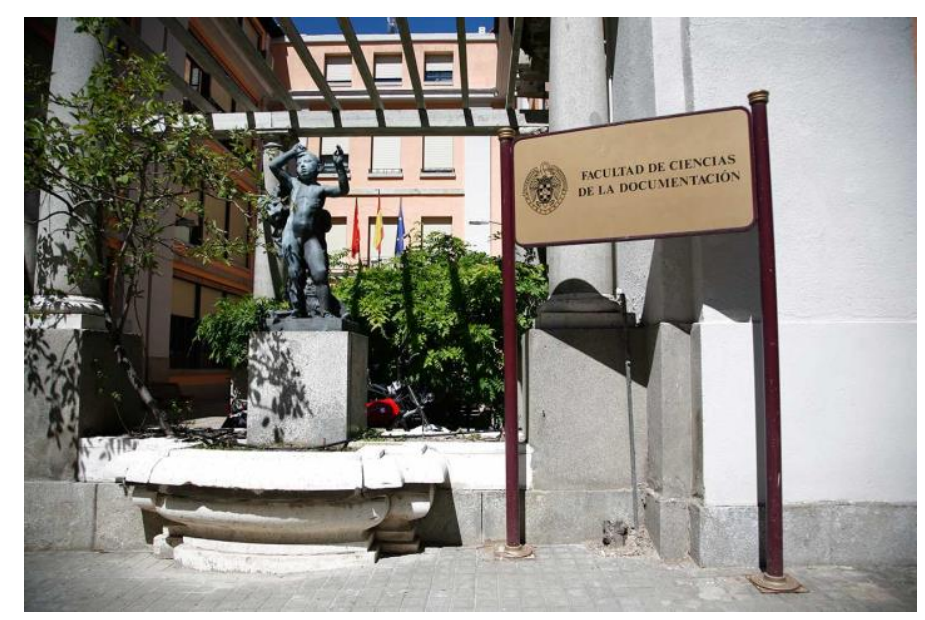

Fig.1 Facultad de Ciencias de la Documentación de la UCM. Foto María Olivera 
La fotografía en la docencia del área de Biblioteconomía y Documentación en las universidades públicas madrileñas. Actividades del Grupo Fotodoc

Por lo que respecta al resto de universidades de la Comunidad de Madrid, en el área que tratamos solo la Universidad Carlos III desarrolló en la Facultad de Humanidades, Comunicación y Documentación una intensa labor expuesta a través de las Jornadas "Imagen, Cultura y Tecnología", de amplio espectro, con la publicación de las Actas de los distintos eventos (2002, 2004, 2005, 2006, 2007). El Grado en Documentación de la Universidad Carlos III no contemplaba asignaturas específicas sobre fotografía. Fue renovado en 2016 y cambió su denominación para ser ofertado en el 2017-2018 por primera vez con el título Grado en Gestión de la Información y contenidos digitales. Su orientación es tecnológica, y la fotografía tampoco está entre las asignaturas ofertadas, si bien puede estudiarse parcialmente en la digitalización de contenidos.

Aunque el carácter transversal de la fotografia la vincula a muchas actividades y obviamente a disciplinas y materias diversas, hasta el momento indicado no se conocen asignaturas específicas sobre fotografía dentro del área salvo las que se han indicado e indican a continuación. De entonces a hoy la oferta no ha variado en la UCM, sino que al contrario los estudios específicos se han reducido ya que al aprobarse el Grado en Información y Documentación (2009) la asignatura pasó a denominarse Documentación Fotográfica y Audiovisual, con un temario amplio y por tanto con menos posibilidades de profundizar en ninguna de las dos disciplinas, lo que obligó a los responsables a complementar los contenidos con actividades paralelas, tales como jornadas, seminarios o encuentros.

Los alumnos matriculados entre 2010 y 2017, es decir en 7 cursos, suman 606, lo que hace una media de más de 85 alumnos por curso (Tabla 1). El descenso en la matrícula fue premeditado, y se debe al cambio en la normativa para limitar el número de alumnos y facilitar así las prácticas, visitas y resto de actividades formativas, de acuerdo a los planes de Bolonia.

Tabla 1. Alumnos matriculados en la asignatura Documentación Fotográfica Grado en Información y Documentación (UCM), 2010-2017

\begin{tabular}{lr}
\hline \multicolumn{1}{c}{ CURSO ACADÉMICO } & ALUMNOS \\
\hline $2010-2011$ & 139 \\
$2011-2012$ & 124 \\
$2012-2013$ & 93 \\
$2013-2014$ & 49 \\
$2014-2015$ & 77 \\
$2015-2016$ & 67 \\
$2016-2017$ & 57 \\
& TotaL \\
\hline
\end{tabular}

Por otra parte, los profesores especializados, con una línea de investigación vinculada a la fotografía, se preocuparon de que ésta tuviera su espacio en asignaturas de las Licenciaturas en Comunicación, Periodismo y 
Publicidad de la Facultad de Ciencias de la Información, tales como Documentación Informativa, Documentación Audiovisual y Documentación publicitaria.

En 2006 se aprobó el Máster en Gestión de la Información de la Facultad de Ciencias de la Documentación, de dos años de duración, reformado en 2010 con cinco especialidades, una de las cuales estaba dedicada a los Medios de Comunicación. Dentro de esa rama se incluyó la asignatura Documentación Fotográfica e Iconográfica, que ha desaparecido al ser reformado de nuevo el Máster y desparecer la especialidad en Medios, por tanto fue impartida oficialmente durante siete cursos entre octubre de 2010 y septiembre de 2017, con una media de 10 alumnos por curso.

Las modificaciones en Máster y Grado suponen que actualmente solo se imparta la asignatura Documentación Fotográfica y Audiovisual, con un nuevo programa que contempla ambos especialidades. EL objetivo fundamental de la asignatura es conocer, gestionar y analizar los documentos fotográficos y audiovisuales. Con este fin, el enfoque es más práctico, en el que se combina la adquisición de conocimiento con prácticas relacionadas con los documentos fotográficos y audiovisuales.

1. Conceptos e historia de la documentación fotográfica y audiovisual

2. Documentación fotográfica. Evolución, tipología y características

3. Documentación audiovisual. Evolución, tipología y características

4. Centros de documentación fotográficos y audiovisuales

5. Análisis documental

6. Funciones del documentalista gráfico

7. Derechos y propiedad intelectual

\section{Grupo de Investigación Fotodoc}

Durante una década la fotografía estudiada como documento fue cogiendo peso específico y a partir del año 2010 Juan Miguel Sánchez Vigil y María Olivera Zaldua crearon en la UCM un Grupo de Trabajo, denominado Fotodoc (Fotografía y Documentación), al que se incorporaron los profesores Juan Carlos Marcos Recio y Antonia Salvador Benítez, todos ellos interesados en distintos aspectos de la documentación fotográfica (recuperación, gestión, tratamiento, análisis y difusión). Esta iniciativa fue germen del Grupo de Investigación Fotodoc, reconocido oficialmente en junio de 2016, con la participación de sus miembros en proyectos relevantes tales como: "Estudio de viabilidad del Plan de Catalogación y Digitalización de los Fondos Históricos conservados en el Instituto Patrimonio Histórico Español (IPHE) con el fin de que puedan integrarse en el Proyecto de la Biblioteca Digital Europea. Aplicación al fondo fotográfico Pando", "La nueva ecología de la información y la documentación en la sociedad del conocimiento: desarrollo de una métrica sistémica, planificación de un observatorio para su seguimiento e identificación de tendencias básicas y retos estratégicos (infoscopos.com)" e "Imágenes del nuevo mundo: el patrimonio artístico portugués e iberoamericano a través del legado fotográfico de Diego Angulo Íñiguez al Consejo Superior de Investigaciones Científicas”.

El Grupo es responsable de las Jornadas del mismo nombre (Fotodoc), cuyo objetivo es acercar a los estudiosos las actividades y tareas que se realizan en centros públicos y privados (bibliotecas, archivos, empresas, instituciones, organizaciones, etc.). Además ha organizado dos Congresos Internacionales. Uno de los trabajos recientes en relación con la Fotografía y los programas docentes de Doctorado son los artículos elaborados por los miembros del grupo sobre las tesis doctorales de fotografía realizadas en España. De estas investigaciones, planteadas en dos periodos resultan los siguientes datos (Tabla 2). 
La fotografia en la docencia del área de Biblioteconomía y Documentación en las universidades públicas madrileñas. Actividades del Grupo Fotodoc

Tabla 2. Temática general de los contenidos de las tesis (1976-2016)

\begin{tabular}{lr}
\hline \multicolumn{1}{c}{ CATEGORÍA } & No DE TESIS $^{\circ}$ \\
\hline Arte & 108 \\
Comunicación & 54 \\
Sociología & 41 \\
Técnica y Tecnología & 48 \\
Documentación & 54 \\
Autores (vida y obra) & 43 \\
Especialización (NUEVO) & 10 \\
Historia & 19 \\
& ToTAL \\
\hline
\end{tabular}

Actualmente, el Grupo Fotodoc desarrolla varios programas de investigación con fondos históricos en centros públicos y privados, entre ellos la Real Academia Española, la Biblioteca Histórica de la Universidad Complutense y el Instituto Valencia de Don Juan. Fruto de estos estudios son los libros: Los daguerrotipos del Instituto de Valencia de Don Juan y Portugal inédito. Fotografias de Eduardo Hernández Pacheco. Así mismo el Grupo realiza exposiciones (Fig. 2 y 3 ) de carácter documental con carácter formativo, en cuyo diseño y montaje colaboran los alumnos, entre ellas las siguientes en los últimos tres años.

1. Estampas de África. Fotografias del capitán médico Jorge Bosch Díaz. Facultad de Ciencias de la Documentación de la UCM (11 de mayo-2 de junio de 2017).

2. Portugal Inédito. Biblioteca Histórica Marqués de Valdecilla (30 de abril-30 de septiembre de 2017).

3. La fotografia en sus reversos. La puerta de atrás. Facultad de Ciencias de la Documentación de la UCM (22 de marzo-7 de abril de 2017).

4. Portugal inédito. Fotografias de Eduardo Hernández-Pacheco. Oporto: Centro Portugués de Fotografía (22 de julio-1 de septiembre de 2016).

5. Fotoimaginando. El universo de la fotografía. XVI Semana de la Ciencia. Facultad de Ciencias de la Documentación de la UCM (7-20 de noviembre de 2016))

6. Cervantes. Variaciones. Madrid. Facultad de Ciencias de la Documentación (28 de abril-13 de mayo de 2016).

7. Los álbumes de Adela Crooke. Fotografias del Instituto de Valencia de Don Juan. Ateneo de Madrid (1830 de abril de 2016).

8. Kaulak. Retratos y paisajes. Ateneo de Madrid (1-15 de marzo de 2016) 


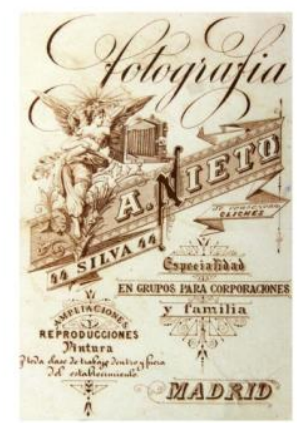

Grupo Fotodoc

(Fotografia y Documentación)

22 de marzo-7 de abril

Facultad de Ciencias de la Documentación
Universidad Complutense de Madrid

Fig.2 Cartel de la Exposición "La fotografia en sus reversos", 2017.

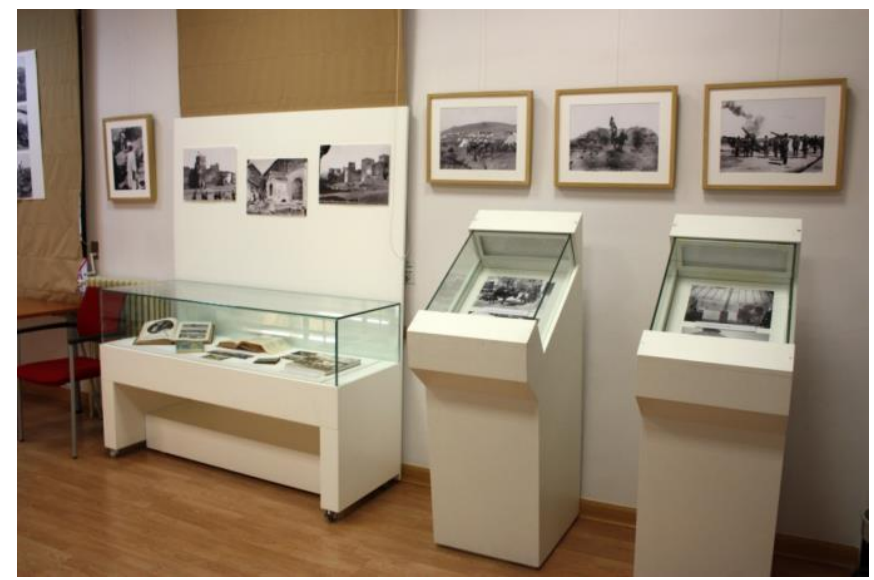

Fig. 3. Exposición Estampas de África, 2017. Foto: María Olivera

\section{Jornadas Fotodoc (Fotografía y Documentación)}

Las Jornadas Fotodoc fueron creadas en 2011 por Juan Miguel Sánchez Vigil y María Olivera Zaldua en la Facultad de Ciencias de la Documentación de la Universidad Complutense, siendo decano de la misma Luis Fernando Ramos Simón (Fig 4). El objeto fue el estudio y difusión dela fotografía desde el punto de vista de la Documentación, es decir desde su valor como documento en el más amplio sentido. Por consiguiente se abrió una vía de trabajo transversal puesto que se contemplaba el análisis de la fotografía en fondo y forma, así como su conservación, tratamiento y difusión en las instituciones responsables de custodia y en todas aquellas colecciones, públicas o privadas, con fondos fotográficos (Tabla 3).

Una vez puesto en marcha el proyecto se montó un equipo de trabajo, activo como Grupo de Investigación con el mismo nombre que las Jornadas desde junio de 2016, del que forman parte Sánchez Vigil, Olivera Zaldua, Antonia Salvador Benítez y Fecderico Ayala Sörensen. En el Grupo de Investigación colaboraron también temporalmente Juan Carlos Marcos Recio y Alicia Arias Coello, actualmente en otros Grupos acordes a sus líneas: Publicidad y Gestión de la Información. 
La fotografía en la docencia del área de Biblioteconomía y Documentación en las universidades públicas madrileñas. Actividades del Grupo Fotodoc

Tabla 3. Jornadas Fotodoc

\begin{tabular}{ll}
\hline \multicolumn{1}{c}{ FECHA } & \multicolumn{1}{c}{ TEMA } \\
\hline $2011 / 04 / 07$ & Fotoperiodismo \\
$2011 / 11 / 23$ & Instituciones y fondos \\
$2012 / 04 / 19$ & Fotografia digital \\
$2012 / 12 / 19$ & Lectura de las imágenes \\
$2013 / 05 / 07$ & Investigación de la fotografía \\
$2013 / 12 / 12$ & La fotografía como objeto de deseo \\
$2014 / 12 / 04$ & Diversidad de los fondos \\
$2015 / 12 / 10$ & Variaciones \\
\hline $2016 / 11 / 10-11$ & Visiones \\
\hline $2017 / 05 / 18$ & Fotografía en la Guerra de África \\
\hline
\end{tabular}

Fuente: Elaboración propia

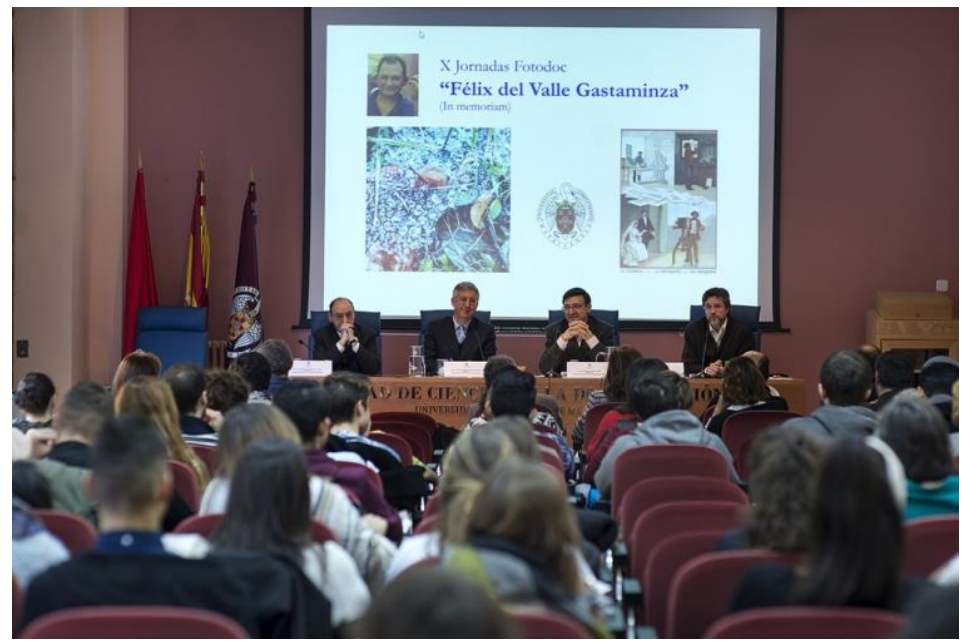

Fig.4 X Jornadas Fotodoc, noviembre de 2016. Foto María Olivera Zaldua

\section{Los Congresos Internacionales de Documentación Fotografía}

En abril de 2014, con el fin de ampliar el campo de estudio e intercambiar experiencias con otros investigadores, el Grupo Fotodoc acometió la tarea de organizar un Congreso Internacional. De acuerdo con diversas instituciones se conformó un comité científico compuesto por miembros de varias universidades y se fijó una periodicidad bienal. Se establecieron dos objetivos fundamentales: difundir los estudios sobre fotografía e intercambiar experiencias entre profesionales, investigadores y estudiantes. 
El primer Congreso se celebró en la Facultad de Ciencias de la Documentación de la Universidad Complutense los días 2, 3 y 4 de abril de 2014. El eje central fue la conmemoración del 175 aniversario de la presentación de la fotografía en París por Daguerre. Participaron instituciones de México (Instituto de Investigación de Estética de la Universidad Nacional Autónoma), Portugal (Centro Portugués de Fotografía de Oporto), Brasil (Universidad de Brasilia) y España (Centre de Recerce i Difusió de la Imatge, CSIC, UCM, Revista Arte Fotográfico, Universidad Carlos III, Politécnica de Valencia, Escuela Universitaria TAI, ABC, Biblioteca Nacional, Instituto Cultural de España y Photomuseum de Zarautz).

El segundo Congreso se desarrolló entre el 7 y el 9 de marzo de 2016 en la Facultad de Ciencias de la Información de la Universidad de San Luis Potosí (México), con la participación del Sistema Nacional de Fototeca (SINAFO), Museo Regional Potosino, Instituto de Investigaciones Estéticas de México, Universidad de San Luis, Centro de Investigaciones Interdisciplinarias en Ciencias y Humanidades, Bienal Internacional del Cartel de México y la Universidad Complutense de Madrid (Fig. 5).

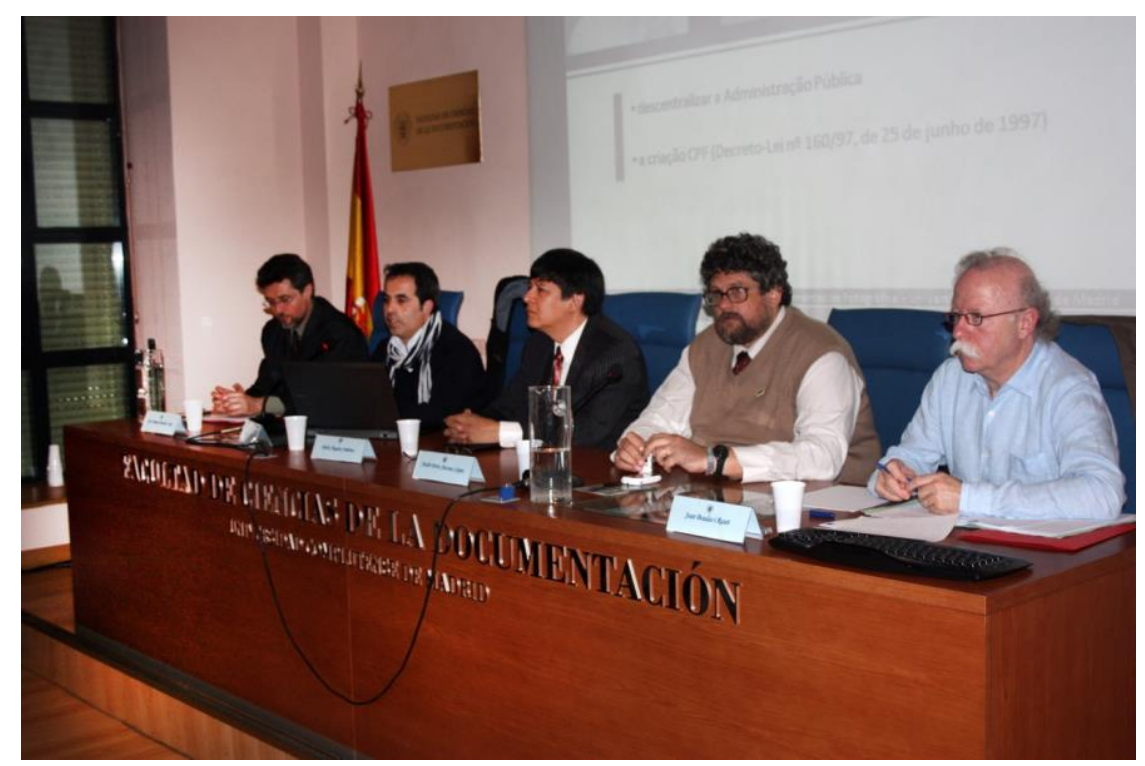

Fig. 5 Primer Congreso Internacional de Documentación Fotográfica celebrado en la Facultad de Ciencias de la Documentación de la UCM. De izquierda a derecha: Juan Miguel Sánchez Vigil (UCM), Bernardino Castro (Centro Portugués de Fotografia), Pedro Ángeles (Instituto de Investigaciones Estéticas de la UNAM, André Porto (Universidad de Brasilia) y Joan Boadas (Centre de Recerca i Difusió de la Imatge, Girona). Foto: María Olivera. 
La fotografía en la docencia del área de Biblioteconomía y Documentación en las universidades públicas madrileñas. Actividades del Grupo Fotodoc

\section{Referencias}

CAeiro Rodríguez, M. (Coordinador, 2017). Descubrir el arte. Formación en artes plásticas y visuales para maestros de Primaria. Logroño: Universidad Internacional de La Rioja.

Olivera Zaldua, M., SÁnchez Vigil, J.M., Marcos Recio, J.C. (2016). “Análisis de las tesis doctorales sobre fotografía en las Universidades españolas (enero de 2013-marzo de 2016)” en Ibersid, 11:2, p. 13-21.

SÁnchez Vigil, J.M. (1999). El Universo de la fotografia. Prensa, Edición, Documentación. Madrid: Espasa.

Sánchez Vigil, J.M., Marcos Recio, J.C., Olivera Zaldua, M. (2014). "Tesis doctorales sobre fotografía en la universidad española. Análisis de la producción y dirección (1976-2012)" en Revista Española de Documentación Cientifica, 37 (1), enero-marzo, p. 1-14.

Sánchez Vigil, J.M, Olivera Zaldua, M.; Salvador Benítez, A. (2015). Los daguerrotipos del Instituto de Valencia de Don Juan. Madrid: UCM.

Sánchez Vigil, J.M, Olivera Zaldua, M.; Salvador Benítez, A. (2016). Portugal Inédito. Fotografias de Eduardo Hernández-Pacheco. Madrid: UCM.

Valle GastaminZa, F. del (Coordinador, 1999). Manual de Documentación Fotográfica. Madrid: Síntesis.

Vega Pérez, C. (2014). "Los estudios de fotografía en la Universidad" en Olivera Zaldua, M, Salvador Benítez, A. Del artefacto mágico al píxel estudios de fotografía. XXIII Jornadas Fadoc: I Congreso Internacional de Documentación Fotográfica (175 aniversario de la fotografia).. Facultad de Ciencias de la Documentación. Universidad Complutense de Madrid 